m37.004

\section{Investigation of dynamic processes in lipid-based nanoparticulate drug carrier systems with small and wide angle $X$-ray diffraction}

\section{Heike Bunjes, Judith Kuntsche, Gert Wörle}

Friedrich-Schiller-Universität Jena, Institute of Pharmacy, Department of Pharmaceutical Technology, Lessingstr. 8, 07743 Jena, Germany

\section{Keywords: nanoparticles, lipids, pharmaceuticals}

Colloidal lipid-based particles offer interesting possibilities in drug delivery, in particular for the administration of poorly water soluble drugs, e.g. into the bloodstream. Such particles can be prepared by high-pressure homogenisation of the respective lipids in an aqueous phase with adequate stabilizers. Since the material properties of the matrix lipids in the colloidal state may differ significantly from those of the raw materials adequate characterization of the prepared particles is a very important part of the development work on such systems to ensure the formation of the desired particle properties. X-ray diffraction (XRD) in combination with complementary methods such as, e.g., differential scanning calorimetry (DSC) and electron microscopy, has proven to be a very effective tool for this purpose. Examples will be shown from three different types of lipid drug carrier systems: solid lipid nanoparticles, supercooled smectic nanoparticles and nanoparticles of cubic phase. When solid lipid nanoparticles are prepared via triglyceride melts, a pronounced supercooling effect is often observed which may impair the formation of the desired solid state. Certain emulsifiers can increase the crystallization temperature and thus improve the solidification behaviour of the nanoparticles but their use is often accompanied by additional thermal events prior to triglyceride crystallization. According to XRD these additional thermal events correspond to the crystallization of a surfactant layer at the particle surface which may also include some of the triglyceride in dependence on the composition. The emulsifiers may also modify the polymorphic transitions of the triglycerides after crystallization. Generally, polymorphic transitions are faster in the nanoparticles than in the bulk and sometimes a more stable modification than observed for the bulk material is reached. Also within the colloidal size range different transition rates can be observed with smaller particles transforming faster than larger ones. For small nanoparticles from saturated monoacid triglycerides, a structured melting event is observed in DSC that is not caused by polymorphism but has to be attributed to a particle size effect according to XRD. An influence of the type of emulsifier is also observed for the crystallization behaviour of nanoparticles based on supercooled smectic phase cholesterol esters. The typical bimodal crystallization process observed in phospholipid-containing cholesteryl myristate dispersions is not due to polymorphism as confirmed by XRD but probably reflects the crystallization properties of particles with different shapes. The temperature dependence of different preparation processes for cubic phase nanoparticles based on aqueous monoolein/poloxamer systems seems to be related to the phase behaviour as indicated by results from small angle XRD. m37.005

\section{Role of co-reactants during the formation of silica gels assessed by in situ SAXS}

\author{
Cédric J. Gommes $^{\text {a }}$, Bart Goderis ${ }^{\mathrm{b}}$, Jean-Paul Pirard ${ }^{\mathrm{a}}$,
} Silvia Blacher ${ }^{\mathrm{a}}$

${ }^{a}$ University of Liège, Department of Chemical Engineering, Belgium, ${ }^{b}$ Catholic University of Leuven, Chemistry Department, Belgium.E-mail: cedric.gommes@ulg.ac.be

\section{Keywords: silica gel, phase separation, SAXS}

The sol-gel process is a versatile way to synthesize porous materials [1]. Pure silica gels, obtained from the polycondensation of tetraethoxysilane (TEOS) in ethanol, collapse completely during evaporative drying [1]. On the contrary, hybrid silica gels synthesized by co-polymerizing TEOS with organically modified trialkoxysilane molecules can withstand evaporative drying. The final porous materials still possess a high porosity, which makes them interesting for many applications, most notably in catalysis [2]. To understand the ability of co-polymerized hybrid silica gels to withstand desiccation, the mechanism by which they form is investigated at the nanometer scale using in situ SAXS. The reported time-resolved data were obtained at DUBBLE, the Dutch-Flemish beamline at the European Synchrotron Radiation Facility in Grenoble, France. The mechanism of formation of the gels switches from the aggregation of colloidal particles for pure silica gels, to a reactioninduced phase separation when a co-reactant is used. The latter process is evidenced by SAXS through the appearance and growth of a scattering maximum, followed by its shift towards smaller scattering angles, which patterns are typical of spinodal decomposition [3].

[1] Brinker, C.J., Scherer, G.W., Sol-Gel Science, the Physics and Chemistry of Sol-Gel Processing, Academic Press: San Diego, 1990. [2] B. Heinrichs, P. Delhez, J.-P. Schoebrechts, J.-P. Pirard, J. Catal., 1997, 172, 322.

[3] C.J. Gommes, S. Blacher, B. Goderis, R. Pirard, B. Heinrichs, C. Alié, J-P. Pirard, J. Phys. Chem. B, 2004, 108, 8983. 